Nonlinear diffusion in two-dimensional ordered porous media based on a free volume theory

A. Godec, M. Gaberscek, J. Jamnik, and F. Merzel

Citation: The Journal of Chemical Physics 131, 234106 (2009); doi: 10.1063/1.3274638

View online: http://dx.doi.org/10.1063/1.3274638

View Table of Contents: http://aip.scitation.org/toc/jcp/131/23

Published by the American Institute of Physics

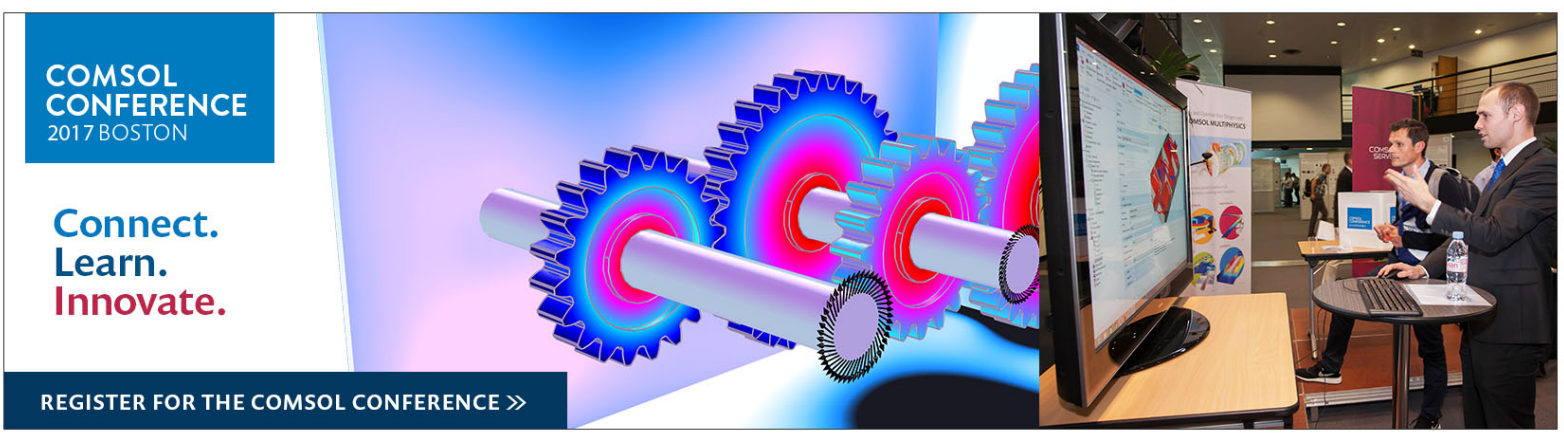




\title{
Nonlinear diffusion in two-dimensional ordered porous media based on a free volume theory
}

\author{
A. Godec, ${ }^{\text {a) }}$ M. Gaberscek, J. Jamnik, and F. Merzel \\ National Institute of Chemistry, Hajdrihova 19, Ljubljana 1000, Slovenia
}

(Received 3 September 2009; accepted 24 November 2009; published online 17 December 2009)

\begin{abstract}
A continuum nonlinear diffusion model is developed to describe molecular transport in ordered porous media. An existing generic van der Waals equation of state based free volume theory of binary diffusion coefficients is modified and introduced into the two-dimensional diffusion equation. The resulting diffusion equation is solved numerically with the alternating-direction fully implicit method under Neumann boundary conditions. Two types of pore structure symmetries are considered, hexagonal and cubic. The former is modeled as parallel channels while in case of the latter equal-sized channels are placed perpendicularly thus creating an interconnected network. First, general features of transport in both systems are explored, followed by the analysis of the impact of molecular properties on diffusion inside and out of the porous matrix. The influence of pore size on the diffusion-controlled release kinetics is assessed and the findings used to comment recent experimental studies of drug release profiles from ordered mesoporous silicates.
\end{abstract}

(C) 2009 American Institute of Physics. [doi:10.1063/1.3274638]

\section{INTRODUCTION}

The importance of understanding molecular transport in inhomogeneous media has long been recognized. Especially diffusion from ordered mesoporous matrices ${ }^{1}$ recently attracted much attention due to potential of such systems to serve as platforms for controlled delivery of biologically active substances (drugs). ${ }^{2}$ Drug release kinetics often determine the physiological action of the administered drug, in terms of magnitude, duration, and often also side effects, therefore one cannot overlook the potential benefits of understanding and altering release mechanisms. Having highly ordered and controllable structure and pore symmetry (see for example schematics in Fig. 1) ordered mesoporous materials may someday replace traditional polymer based systems.

Theoretical attempts to model diffusion in porous media are based either on percolation theory, ${ }^{3-5}$ computer simulations (molecular dynamics and lattice Monte Carlo), ${ }^{4-11}$ simple continuum models, ${ }^{12}$ and nonlinear diffusion theory. ${ }^{13}$ Due to the limitation to nanosecond timescales, atomic detail simulations cannot be employed to problems of diffusion from ordered porous media, which take place on a secondto-hour timescale. Macroscopic approaches to model linear diffusion from porous media have also been reported. Laudone et al. ${ }^{14}$ used a semianalytical treatment of transport assuming linear gradients between pore chambers and onedimensional diffusion inside chambers. Lemaire et al. ${ }^{15}$ modeled linear diffusion from an erodible polymer based porous system. They modeled the pores as idealized cylinders with time dependent radius, to take into account the pore size variation due to matrix erosion and focused on the evolution of the concentration outside the pore. Herein we attempt to

${ }^{\text {a)} E l e c t r o n i c ~ m a i l: ~ a l j a z . g o d e c @ k i . s i . ~}$ model nonlinear transport from model ordered porous structures with the focus on the evolution of concentration field both inside and outside the porous matrix.

The objective to model such systems is obviously a continuum model but with a firmly established connection to some microscopic molecular properties. In the present work we use a free volume theory of diffusion coefficients of binary simple liquids based on the generic van der Waals (vdW) equation of state for liquid mixtures, ${ }^{16}$ which, as we shall demonstrate, enables the incorporation of molecularscale properties into the macroscopic nonlinear diffusion equation. In the theory of Rah and $\mathrm{Eu},{ }^{16}$ the connection between molecular properties (i.e., interaction potentials) and transport properties is introduced by the determination of free volume from a generic vdW equation of state. In continuum nonlinear diffusion theory usually some mathematically convenient ansatz is used to describe the concentration dependence of the diffusion coefficient ${ }^{17}$ which often does not include any formal physical connection to the concentration dependent microscopic dynamics.

Here the concentration dependence of the diffusion coefficients is established through the relation between free volume and the generic vdW equation of state, which we simplify using well known mixing rules from the general $\mathrm{vdW}$ theory. This approximation is, of course, rather arbitrary but we assume that it should hold reasonably well for not too asymmetric systems solute-solvent. Furthermore we are not interested in the exact values of generic vdW parameters and the resulting diffusion coefficients as Rah and $\mathrm{Eu},{ }^{16}$ but merely in the general behavior of nonlinear mass transport from porous media and the differences brought about by a small variation in molecular parameters.

In order to obtain analytically integrable equations for the concentration dependent diffusion coefficient in the case of particular molecular properties, which are required in the 


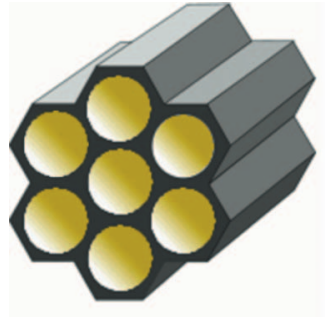

(a)

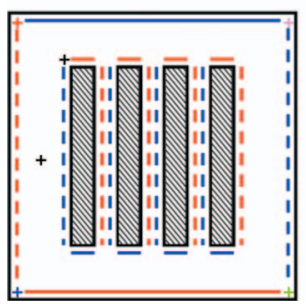

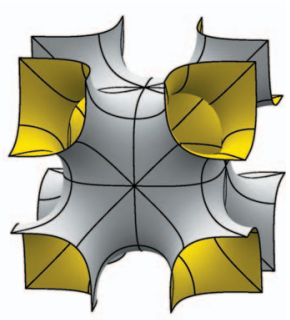

(b)

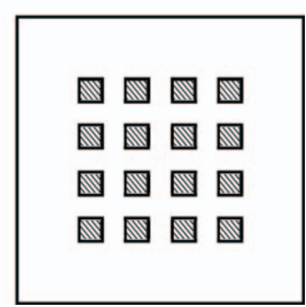

(c)

FIG. 1. Schematics of (a) hexagonal porous matrix such as SBA-15 and (b) I-WP minimal surface which represents the pore configuration of cubic mesoporous materials such as SBA-16. (c) Schematics of 2D models of (a) and (b), where the shaded regions represent impenetrable pore walls. The four types of colored lines and five colored plus signs represent different types of grid points requiring its own formulation of the diffusion Eqs. (10) and (11). Note that the black plus signs represent ordinary grid points, which we find at sites having all four nearest neighbors. The particle and pore sizes are not in scale.

numerical algorithm, two commonly used types of pore symmetries are considered, hexagonal and cubic (Fig. 1). Both systems are modeled as two-dimensional (2D) networks with perfectly smooth impenetrable walls (shaded regions) and constant pore sizes. For a review of the aspects of modulated pore geometry on the diffusion coefficient, see, for example, Ref. 18. The hexagonal symmetry is modeled as parallel channels of finite length while in the case of cubic symmetry an equal number of parallel channels is additionally placed perpendicularly, creating an interconnected network (Fig. 1). The latter should mimic the interconnection of pores in cubic systems and correspond approximately to taking the diagonal plane in the unit cell of the so called $I-W P$ minimal surface, which accurately describes the symmetry of SBA-16 type materials. ${ }^{19}$ The actual ordered mesoporous particle properties are described by means of geometrical factors. In both cases the porous network [shown in Figs. 1(a) and 1(b)] is assumed to extend periodically throughout the entire particle. The particle size can be varied experimentally, but is always much larger than the pore diameter, which typically lies in the range $5-50 \mathrm{~nm}$, depending on the actual material. While the hexagonal system is well described by means of a $2 \mathrm{D}$ parallel slit model (taking into account two principal diffusion directions in a cylindrical pore, radial and axial) the simplification of the cubic symmetry as being a network of square voids connected with slits is somewhat more drastic (in the sense that one effectively loses one possible diffusion direction) so that only qualitative findings can be extracted from the cubic model. In all cases a buffer zone is modeled around the porous particle, describing the surrounding solution in into which the model substance diffuses. The evolu- tion of the concentration distribution is calculated by numerically solving the 2D nonlinear diffusion equation by means of the operator splitting concept under Neumann boundary conditions.

\section{THE DIFFUSION COEFFICIENT}

Throughout the work we assume that the diffusion coefficient does not explicitly depend on pore size. The most common interpretation of molecular diffusion in a simple model liquid is the one of molecules jumping into nearby free volume regions created by density fluctuations. Such reasoning is the basis for free volume theories. ${ }^{20}$ However, it is not possible to determine the free volume within the framework of these simple theories. Recently, Rah and $\mathrm{Eu}^{21}$ used the generic vdW equation of state to define the free volume and diffusion coefficients for binary mixtures. According to their approach the binary diffusion coefficient can be written as ${ }^{16}$

$$
D_{12}=D_{12}^{0} \exp \left(-v_{12}^{c} / v_{f}\right)
$$

where $D_{12}^{0}$ represents the Chapman-Enskog formula for the diffusion coefficient. ${ }^{22}$ The exponential term describes the distribution of voids in the binary mixture, where $v_{12}^{c}$ stands for the common characteristic volume for the two species of the mixture, while $v_{f}$ represents the free volume per molecule. $v_{12}^{c}$ is defined as the mean value of molecular volumes: $v_{12}^{c}=v_{1}^{0} X_{1}+v_{2}^{0} X_{2}$, where $v_{1}^{0}$ and $v_{2}^{0}$ stand for the molecular volumes of constituent molecules $\left(v_{i}^{0}=\pi \sigma_{i}^{3} / 6\right.$, and $\sigma$ is defined in Appendix A) and $X_{1}$ and $X_{2}$ for the mole fractions given in terms of number densities, $\rho_{i},\left[X_{i}=\rho_{i} /\left(\rho_{1}+\rho_{2}\right)\right]$. The free volume per molecule is expressed by $v_{f}=v(1-B \rho)$, where $v$ represents the specific volume, $v=1 / \rho$, and $B$ the generic vdW coefficient (the term generic is used to note that it results from the statistical-mechanical virial equation of state). The concentration dependence of the diffusion coefficient in Eq. (1) is introduced through the solute mole fraction, while molecular properties are included through molecular volumes and the vdW coefficient $B$. Rah and $\mathrm{Eu}^{16}$ used statistical mechanical expressions to calculate vdW parameters. Since we are interested only in general nonlinear macroscopic diffusion and relative differences in diffusion profiles brought about by the variation of molecular parameters (i.e., relative molecular volumes and the coefficient $B$ ) we use a slightly different approach. We replace the binary mixture concept by the two-component-one-fluid classical $\mathrm{vdW}$ approximation (for a derivation and justification see Appendix A). Thus we write $B$ as

$$
B=X_{1}^{2} B_{11}+2 X_{1} X_{2} B_{12}+X_{2}^{2} B_{22},
$$

where $B_{i i}$ represents the generic vdW coefficient of pure component $i$ (1 stands for the solvent and 2 for the solute) and we assumed $B_{12}=B_{21}$ for the cross coefficient (see Appendix A). The one-fluid approximation is valid for systems where $B_{11}$ and $B_{22}$ do not differ appreciably. ${ }^{23}$ Using Eq. (2) and combining rules for the cross coefficient $\left(B_{12}\right.$ $=\sqrt{B_{11} B_{22}}$ ) we can rewrite Eq. (1) in the form 


$$
D_{12}=D_{12}^{0} \exp \left(\frac{C\left(1-\varphi\left(1+v_{2}^{0} / v_{1}^{0}\right)\right)}{\rho B_{11}\left(1-\varphi\left(1-\sqrt{B_{22} / B_{11}}\right)\right)^{2}-1}\right)
$$

where $C=v_{1}^{0} / v$ is the relative specific volume and we replaced $X_{2}$ with $\varphi$ (the mole fraction of solute) and used $X_{1}$ $=1-\varphi$. Equation (3) describes the diffusion coefficient as a function of solute mole fraction and two molecular parameters, the ratios of molecular volumes, and vdW coefficients. $B$ and $v_{i}$ depend on both attractive and repulsive intermolecular interactions (for details see Appendix A). As we shall see later on, the diffusion coefficient needs to be analytically integrable with respect to the fraction of solute for the developed algorithm. Equation (3), its Taylor expansion or expansion in Padé approximants, is not analytically integrable with respect to $\varphi$, therefore we evaluate Eq. (3) using desired molecular parameters and afterward fit them using the following ansatz:

$$
D_{12} \rightarrow D(\varphi)=a_{1 k} \exp \left(a_{2 k} \varphi\right)+b_{1 k} \exp \left(b_{2 k} \varphi\right)+c_{k} \varphi^{2},
$$

where $k$ stands for the index of the values of molecular parameters (see text below). There is no explicit dependence of the diffusion coefficient on pore size. The dependence is established via differences in local composition, which are directly related to pore network properties (i.e., pore diameter).

\section{NONLINEAR DIFFUSION MODEL AND NUMERICAL ALGORITHM}

Our goal here is to construct a numerical method to describe nonlinear diffusion in ordered porous media using diffusion coefficients derived from a microscopic theory. We describe the evolution of the solute concentration distribution inside and outside the porous matrix with impenetrable hard walls using the $2 \mathrm{D}$ nonlinear diffusion equation

$$
\frac{\partial \varphi}{\partial t}=\frac{\partial}{\partial x}\left(D(\varphi) \frac{\partial \varphi}{\partial x}\right)+\frac{\partial}{\partial y}\left(D(\varphi) \frac{\partial \varphi}{\partial y}\right),
$$

where $\varphi$ stands for the fraction of solute molecules and $D(\varphi)$ for the solute fraction dependent diffusion coefficient. For practical reasons, we introduce a new variable $\psi, \partial \psi / \partial \varphi$ $=D(\varphi)$, so that Eq. (1) can be rewritten as

$$
\frac{\partial \varphi}{\partial t}=\frac{\partial^{2} \psi}{\partial x^{2}}+\frac{\partial^{2} \psi}{\partial y^{2}},
$$

and $\psi$ is obtained by integration of Eq. (4).

For the construction of the numerical algorithm, we use the concept of operator splitting, more specifically the alternating-direction implicit method (ADI) (Ref. 24) according to which we divide each time step into two substeps each treating one of the spatial dimensions implicitly. The finite difference counterparts of Eq. (6) are written as

$$
\begin{aligned}
\varphi_{i, j}^{n+1 / 2}-\varphi_{i, j}^{n}= & \frac{\Delta t}{2 \Delta^{2}}\left(\psi_{i+1, j}^{n+1 / 2}-2 \psi_{i, j}^{n+1 / 2}+\psi_{i-1, j}^{n+1 / 2}+\psi_{i, j+1}^{n}\right. \\
& \left.-2 \psi_{i, j}^{n}+\psi_{i, j-1}^{n}\right),
\end{aligned}
$$

$$
\begin{aligned}
\varphi_{i, j}^{n+1}-\varphi_{i, j}^{n+1 / 2}= & \frac{\Delta t}{2 \Delta^{2}}\left(\psi_{i, j+1}^{n+1}-2 \psi_{i, j}^{n+1}+\psi_{i, j-1}^{n+1}+\psi_{i+1, j}^{n+1 / 2}\right. \\
& \left.-2 \psi_{i, j}^{n+1 / 2}+\psi_{i-1, j}^{n+1 / 2}\right),
\end{aligned}
$$

where $\Delta x=\Delta y=\Delta$ is the grid spacing. We now expand each $\psi$ on the right hand side of Eqs. (7) and (8) to the first order in $\varphi$ and obtain, for example,

$$
\begin{aligned}
\psi_{i, j}^{n+1 / 2} & =\psi_{i, j}^{n}+\left.\left(\varphi_{i, j}^{n+1 / 2}-\varphi_{i, j}^{n}\right) \frac{\partial \psi}{\partial \varphi}\right|_{i, j} \\
& =\psi_{i, j}^{n}+\left(\varphi_{i, j}^{n+1 / 2}-\varphi_{i, j}^{n}\right) D\left(\varphi_{i, j}^{n}\right),
\end{aligned}
$$

where $D(\varphi)$ is given by Eq. (4). The subscripts $i$ and $j$ refer to the grid point numbering along the $x$ and $y$ dimensions, respectively, and the superscript refers to the time step. We can now rewrite Eqs. (7) and (8) as

$$
\begin{aligned}
&-\alpha D\left(\varphi_{i-1, j}^{n}\right) \varphi_{i-1, j}^{n+1 / 2}+\left[1+2 \alpha D\left(\varphi_{i, j}^{n}\right)\right] \varphi_{i, j}^{n+1 / 2}-\alpha D\left(\varphi_{i+1, j}^{n}\right) \varphi_{i+1, j}^{n+1 / 2} \\
&= \alpha\left(\psi_{i-1, j}^{n}+\psi_{i, j-1}^{n}-4 \psi_{i, j}^{n}+\psi_{i+1, j}^{n}+\psi_{i, j+1}^{n}-D\left(\varphi_{i-1, j}^{n}\right) \varphi_{i-1, j}^{n}\right. \\
&\left.+\left[1 / \alpha+2 D\left(\varphi_{i, j}^{n}\right)\right] \varphi_{i, j}^{n}-D\left(\varphi_{i+1, j}^{n}\right) \varphi_{i+1, j}^{n}\right) \\
&-\alpha D\left(\varphi_{i, j-1}^{n+1 / 2}\right) \varphi_{i, j-1}^{n+1}+\left[1+2 \alpha D\left(\varphi_{i, j}^{n+1 / 2}\right)\right] \varphi_{i, j}^{n+1}-\alpha D\left(\varphi_{i, j+1}^{n+1 / 2}\right) \varphi_{i, j+1}^{n+1} \\
&=\alpha\left(\psi_{i-1, j}^{n+1 / 2}+\psi_{i, j-1}^{n+1 / 2}-4 \psi_{i, j}^{n+1 / 2}+\psi_{i+1, j}^{n+1 / 2}+\psi_{i, j+1}^{n+1 / 2}\right. \\
&-D\left(\varphi_{i, j-1}^{n+1 / 2}\right) \varphi_{i, j-1}^{n+1 / 2}+\left[1 / \alpha+2 D\left(\varphi_{i, j}^{n+1 / 2}\right)\right] \varphi_{i, j}^{n+1 / 2} \\
&\left.-D\left(\varphi_{i, j+1}^{n+1 / 2}\right) \varphi_{i, j+1}^{n+1 / 2}\right)
\end{aligned}
$$

where $\alpha=\Delta t /\left(2 \Delta^{2}\right)$. It is immediately recognized that Eqs. (10) and (11) represent tridiagonal sets of coupled linear equations, $\mathbf{T} \vec{\varphi}=\vec{r}$, with the diagonal elements of $\mathbf{T}$ equal to $1+2 \alpha D\left(\varphi_{i, j}^{n}\right)$ and off-diagonal $-\alpha D\left(\varphi_{i-1, j}^{n}\right)$ and $-\alpha D\left(\varphi_{i+1, j}^{n}\right)$ [analogously for Eq. (11)], which have to be solved each step for all values of $j$ and $i$. The grid topology of our system requires to distinguish among nine different types of grid points according to the boundary conditions: points in the corners of the grid and points neighboring edges and pore walls [see Fig. 1(c) for details]. Accordingly, there are several tridiagonal systems of equations of type (10) or type (11) (see Appendix B), which we solve sequentially at each time step. The physical meaning of the Neumann boundary conditions at pore walls and outer boundaries is the following. We model the pore walls as impenetrable, thus by using Neumann conditions we assure that there is no effective driving force for diffusion into the walls. The fact that there is only a hard repulsion with the fluid mixture means that the concentration directly at the wall is influenced only by the concentration gradients in the liquid phase [note that since we are using a macroscopic approach the oscillations of concentration (i.e., density) of both components on the molecular scale which are due to the presence of the wall are entirely neglected].

\section{NUMERICAL RESULTS AND DISCUSSION}

\section{A. The diffusion coefficient}

In applying Eq. (3) for the determination of the diffusion coefficient, we keep in mind the restriction on the validity of the two-component-one-fluid approximation $\left(B_{11}\right.$ and $B_{11}$ 
TABLE I. Expansion parameters used in the calculations.

\begin{tabular}{cccccccc}
\hline \hline$k$ & $v_{2}^{0} / v_{1}^{0}$ & $B_{22} / B_{11}$ & $a_{1 k}$ & $b_{1 k}$ & $a_{2 k}$ & $b_{2 k}$ & $c_{k}$ \\
\hline 1 & 0.5 & 2.0 & 0.2896 & 0.9939 & -2.4889 & -0.0756 & 0.0 \\
2 & 0.5 & 1.0 & 0.6425 & 0.6415 & -0.3726 & -0.3774 & 0.0 \\
3 & 0.5 & 0.5 & 1.2764 & 0.0 & -0.1227 & 0.0 & -0.4042 \\
4 & 1.0 & 2.0 & 0.3319 & 0.9514 & -2.6073 & -0.0896 & 0.0 \\
5 & 1.0 & 1.0 & 1.2840 & 0.0 & -0.5000 & 0.0 & 0.0 \\
6 & 1.0 & 0.5 & 1.2807 & 0.0 & -0.2813 & 0.0 & -0.4529 \\
7 & 2.0 & 2.0 & 0.8763 & 0.4066 & -0.1215 & -2.8321 & 0.0 \\
8 & 2.0 & 1.0 & 0.6420 & 0.6420 & -0.7465 & -0.7535 & 0.0 \\
9 & 2.0 & 0.5 & 1.2955 & 0.0 & -0.6552 & 0.0 & 0.0 \\
\hline \hline
\end{tabular}

must not differ appreciably). Therefore, we choose cases where the volume of the solute molecule is twice as large as, equal to, and two times smaller than the volume of the solvent molecule. As regards ratios $B_{22} / B_{11}$ we are interested in values 2,1 and 0.5 . We consider all possible combinations of these chosen molecular parameters and index them as given in Table I. The remaining parameters $C, B_{11}$, and $\rho$ correspond to molecular properties of the solvent and the total number density. Since we are not interested in a particular solvent nor in the influence of total number density, they represent free parameters. For the sake of simplicity we choose $C$ to be equal to unity. The diffusion coefficient from the Chapman-Enskog formula $D_{12}^{0}$ depends only on the total packing fraction, more precisely on the value of the radial distribution function at contact, which can be described with the use of the Mansoori extension of the Carnahan-Starling equation for hard-sphere mixtures ${ }^{25}$ through the effective hard sphere diameter of the mixture and the constituent number densities. Since we are interested in systems, where solvent and solute particles exhibit similar properties, we may suppose that the effective diameter of the mixture will not differ appreciably from the diameters of the constituents. We are considering softer continuous potentials [i.e., the Lennard-Jones (LJ) potential] and Ben-Naim ${ }^{26}$ has shown that for LJ mixtures the contact values of all radial distribution functions are almost independent of composition (see also Appendix A), thus the term $D_{12}^{0}$ in Eq. (3) is to a very good approximation a constant. For the sake of simplicity we set it equal to 1, and express the diffusion coefficient in Eqs. (3) and (4) in units of $D_{12}^{0}$. Since we are focused in a general qualitative picture of transport in ordered porous media, $\alpha$ and $\Delta$ are free parameters. However due to the fact that we use a continuum description $\Delta$ must be larger that the wavelength of density fluctuations on the molecular scale. The ratios pore-wall thickness/pore size, however, correspond to realistic situations. After testing for several values the product $\rho B_{11}$ (which determines the sensitivity of the diffusion coefficient to compositional changes) was set equal to 5 for numerical convenience. On one hand, the choice is made to exclude singularities at zero solute mole fraction, on the other hand, such a choice ensures the optimal compromise between numerical stability (i.e., round-off error) and sensitivity to compositional changes.

The concentration dependence of the diffusion coefficient with the use of parameters of Table I is shown in Fig. 2. Note that that shapes of the curves switch their character from convex to concave when the ratio $B_{22} / B_{11}$ varies from large to small values. The meaning of the aforementioned variation of parameters and the resulting physical consequences are the following. Given that the repulsive part of the pair potential originates from the overlap of electronic orbitals, a variation of $B$ (or the ratio $B_{11} / B_{22}$, respectively) while keeping the molecular volume (or volumes, respectively) fixed corresponds to a variation of attractive interactions. Thus lowering of $B$ at constant molecular volume is related to an increase in intermolecular attraction. In this respect, for example, going from $k=1$ to $k=3$ in Table I corresponds to increasing attractive interactions between solute particles while keeping the effective molecular volumes of both constituents fixed. Such a variation increases the sensitivity of the diffusion coefficient to compositional variations at higher solute fractions (see Fig. 2) and decreases the diffusion coefficient at higher solute fractions (which is expected). Meanwhile in the case where the attractive interactions between solvent molecules are larger, the diffusion coefficient is more sensitive to compositional variations at low solute fractions.

\section{B. General characteristics of the evolution of concentration distribution}

First we consider systems where initially the solute particles are located at the intersections of pore channels of a

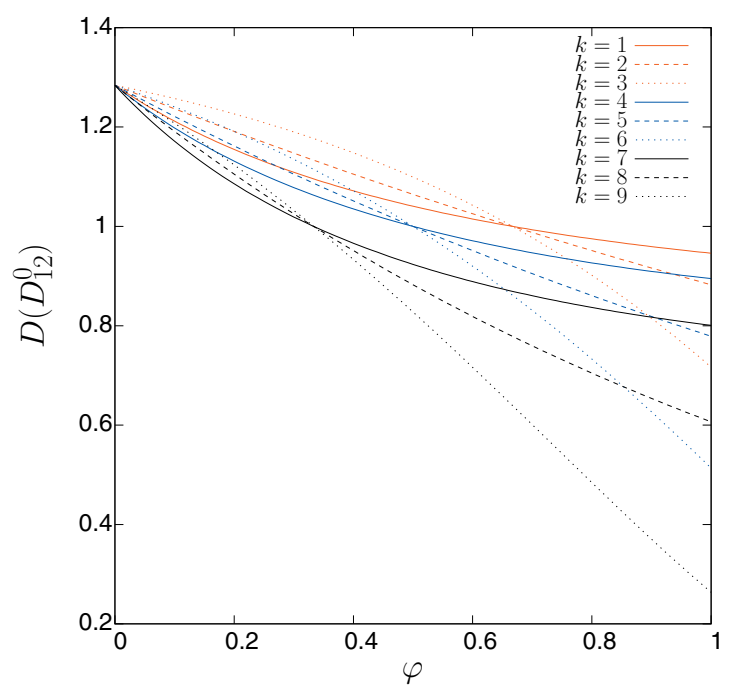

FIG. 2. Concentration dependence of diffusion coefficient from Eq. (4) using the parameters of Table I. 


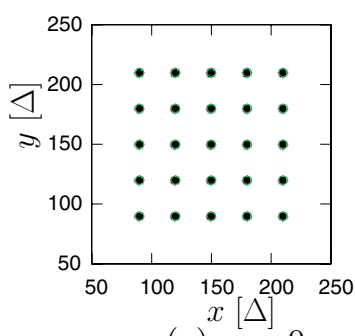

(a) $\tau=0$

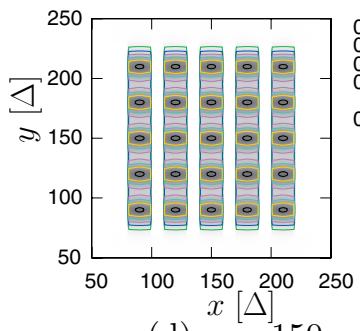

(d) $\tau=150$

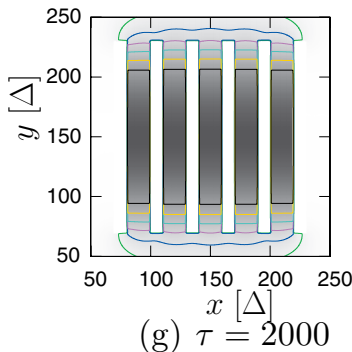

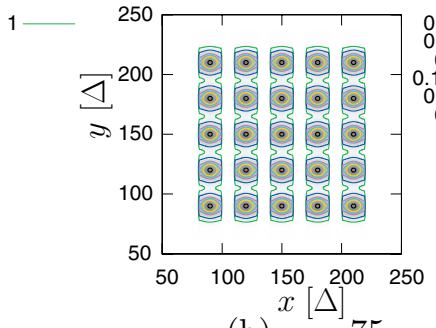

(b) $\tau=75$

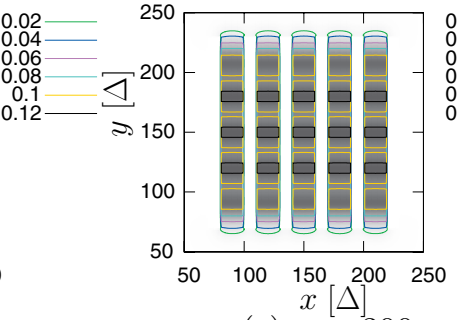

(e) $\tau=300$

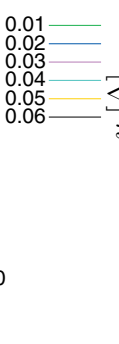

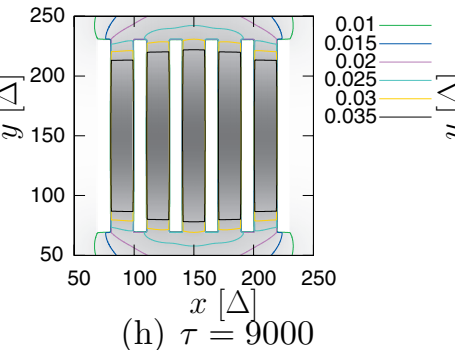

(h) $\tau=9000$

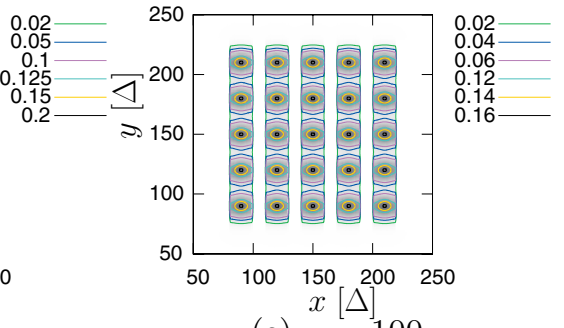

(c) $\tau=100$

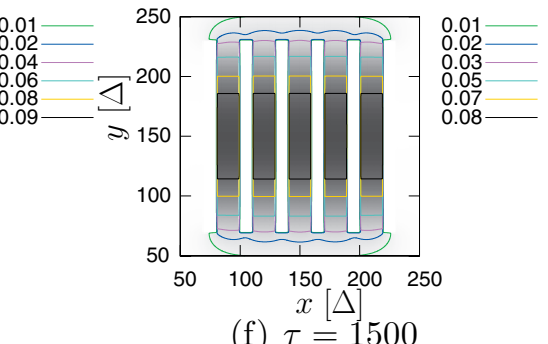

(f) $\tau=1500$

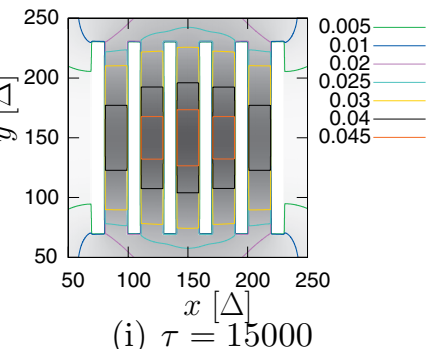

FIG. 3. Contours of $\varphi(x, y)$ after various time intervals in case of hexagonal pore symmetry. The spatial units are those of grid the spacing $\Delta$ and $\tau$ has units of the time step $\Delta t=2 \alpha \Delta^{2}$.

matrix with a cubic symmetry. The thickness of pore walls is set to $10 \Delta$, the pore diameter to $20 \Delta$, and the radius of particles to $4 \Delta$. We modeled the liquid surrounding the porous matrix as an (initially) empty region of width $70 \Delta$ around the porous particle. For the matrix with hexagonal symmetry, the particle and pore wall coordinates and sizes were set in the same way as in case of the cubic symmetry, but in the absence of horizontal pores. For the sake of simplicity we set $D_{12}^{0}=1$. The time evolution of the concentration fields corresponding to the parameter set $k=2$ is shown in Figs. 3 and 4. In both cases it is observed that the concentration fields of individual particles are strongly coupled. For example, diffusion is slower in the interior of the matrix due to the mutual interaction of diffusion layers. This coupling is not limited to diffusion within individual pore channels which is readily observed in Figs. 3 and 4 [see for example Figs. 3(h), 3(i), and $4(\mathrm{~g})-4(\mathrm{i})]$. Of course the strength of interchannel coupling depends on the interpore distance and vanishes for large distances. Thus the single channel model of Lemaire et al. ${ }^{15}$ may not be appropriate for systems with narrow pore walls. Time dependent pore size, as introduced by Lemaire et al., could easily be implemented into the present model.

\section{Impact of molecular properties on the diffusion profiles}

The influence of molecular properties introduced into the expression for the diffusion coefficient was studied in a straightforward manner, by solving the evolution of the model system for all $k$ values given in Table I. Here the porous matrix was built as follows. Similar to section $B$ there is an empty region surrounding the porous matrix particle of width $95 \Delta$. The pore wall thickness and pore diameter were both set to $10 \Delta$ and the radius of particles was set to $4 \Delta$. The concentration distribution along the center of the central pore channel [that is, the line with coordinates $(150 \Delta, y)$ ] of the cubic model matrix was calculated at different times. The results are given in Figs. 5(c) and 5(d). For comparison we plotted also the results of corresponding linear diffusion [Figs. 5(a) and 5(b)] with average diffusion coefficients defined as $\bar{D}_{k}=\int_{0}^{1} D_{k}(\varphi) d \varphi$. It has to be noted that linear diffusion can occur only at very high dilution, since the free volume depends on composition and molecular properties. As observed, the various studied nonlinear systems exhibit almost no difference at very early stages $(\tau<5)$. For $\tau>5$ the differences become progressively more pronounced up to $\tau$ $\approx 75$ and afterward progressively diminish until $\tau \approx 300$. However, they never become significant in case of nonlinear diffusion. In case of linear diffusion, however, the differences are growing progressively so that they become in fact significant for $\tau>150$. We see that the linear theory misleadingly predicts significant differences in concentration profiles inside the porous matrix. The nonlinear diffusion effects obviously antagonize the differences in molecular properties. Naturally, the concentration at the position of particles decreases fastest for the linear and nonlinear diffusion model, in the case where the solute molecules are smaller than those of the solvent and slowest in the reversed case. Overall, the diffusion in the system $k=3$ is fastest while in the $k=8$ sys- 


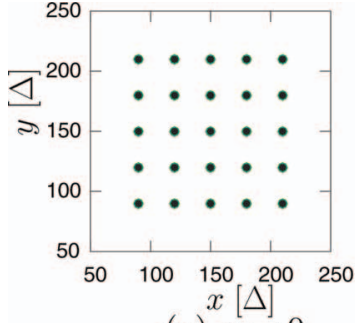

(a) $\tau=0$

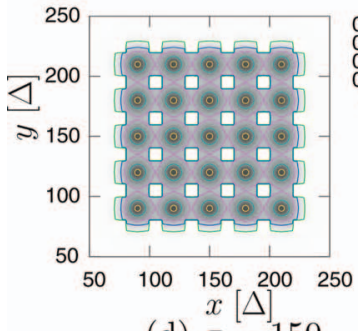

(d) $\tau=150$

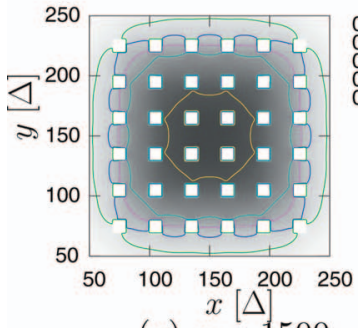

(g) $\tau=1500$

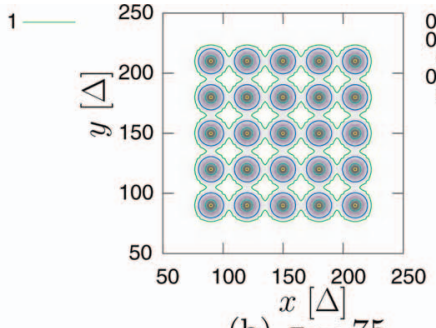

(b) $\tau=75$

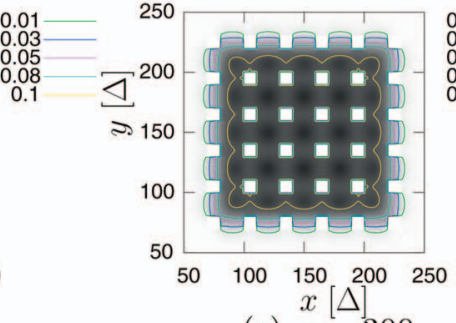

(e) $\tau=300$

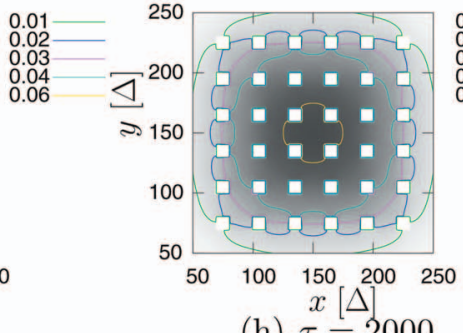

(h) $\tau=2000$

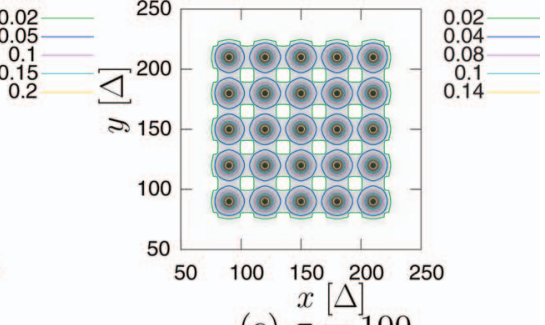

(c) $\tau=100$
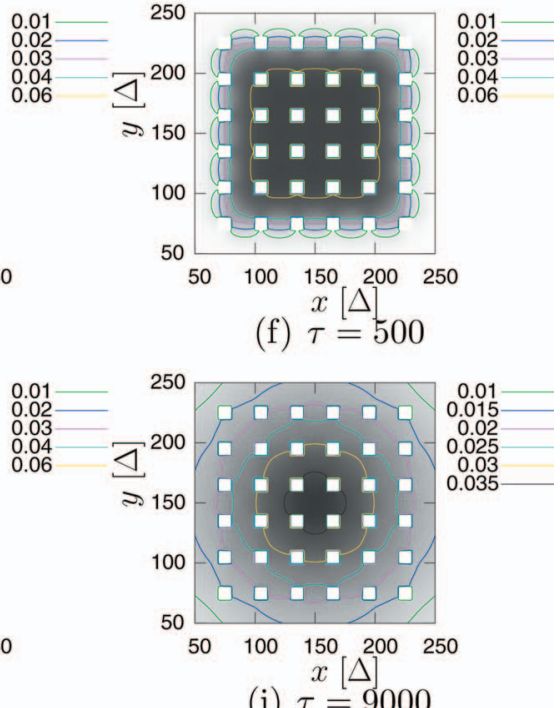

(i) $\tau=9000$

FIG. 4. Contours of $\varphi(x, y)$ after various time intervals in case of cubic pore symmetry. The spatial units are those of grid the spacing $\Delta$ and $\tau$ has units of the time step $\Delta t=2 \alpha \Delta^{2}$.

tem is the slowest. Although the differences due to variation of molecular parameters are very small in the nonlinear case, they turn out to be more pronounced when the solute molecule is larger. Furthermore, differences in molecular parameters in both cases seem to have almost no effect on the concentration profile outside the matrix, that is, diffusion from the matrix seems to be completely independent of molecular properties of the solute. The latter is not surprising if one recognizes that the concentration outside the matrix never reaches high values and the differences in diffusion coefficients become vanishingly small at low solute concentrations. Thus the strategy of controlling the diffusion kinetics by doping solutes into ordered porous matrices can in principle be viewed as quite general and does not depend (at least not strongly) on the particular solute as long as there are no strong interactions between the solute molecule and the walls. Note that for both models (linear and nonlinear) positions where the concentration peaks at short times (due to the position of particles) become depleted at longer times, due to the fact that they correspond to crossing points of horizontal and vertical channels.

\section{Transport from the porous matrix as a function of pore size}

Regarding drug release, one is interested in the variation of the concentration outside the porous matrix. In real systems, the matrix particles in a suspension are themselves subjected to Brownian motion and may be additionally driven by mixing. Effectively this means that there exists a distribution of fluid velocity away from the particle. Assuming no-slip boundary conditions at the particle surface, there must exist a region around the matrix particle, where solute motion is governed purely by molecular diffusion. However at larger distances away from the particle surface one must assume that it is governed by fluid flow. Since the size of the diffusion controlled region depends on particle size, fluid viscosity, temperature, etc., it is most convenient to consider the rate at which solute molecules leave the matrix (i.e., the transfer rate across the outer surface of the porous matrix particle) and how this rate is influenced by the pore size. The local molecular flux across the unit surface at position $\vec{r}$ of the matrix particle can be written as

$$
\vec{j}(\vec{r})=-D(\varphi(\vec{r})) \nabla \varphi(\vec{r}) .
$$

To obtain an expression for the total rate at which molecules exit the matrix, one has to integrate Eq. (12) over the outer surface

$$
\frac{\partial \varphi_{\mathrm{ex}}}{\partial t}=\int_{S} \vec{j}(\vec{r}) \cdot d \vec{S}=-\int_{S} D(\varphi) \nabla \varphi \cdot d \vec{S},
$$

where $\vec{S}=\vec{n} d S$ and $\vec{n}$ is the normal. The cumulative amount of released molecules (which is conventionally measured in experiments) is thus obtained by integration of Eq. (13): 


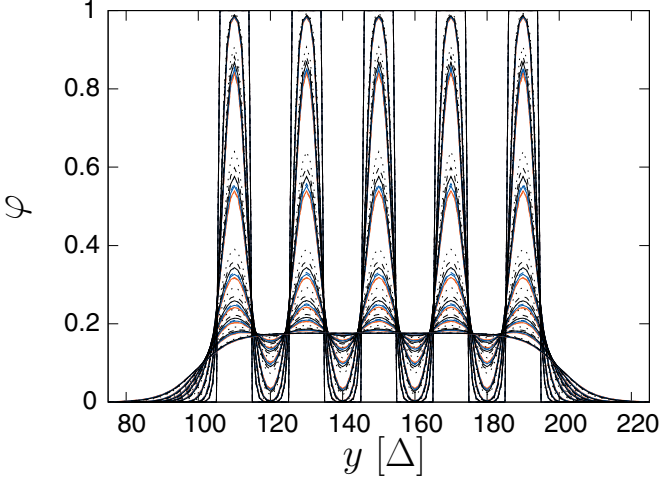

(a)

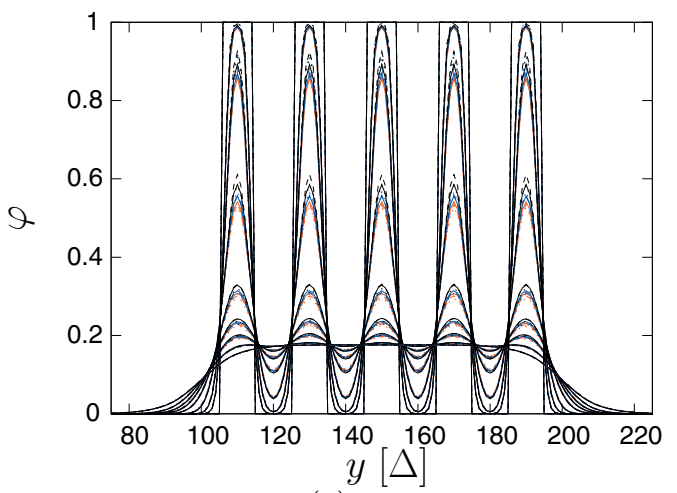

(c)

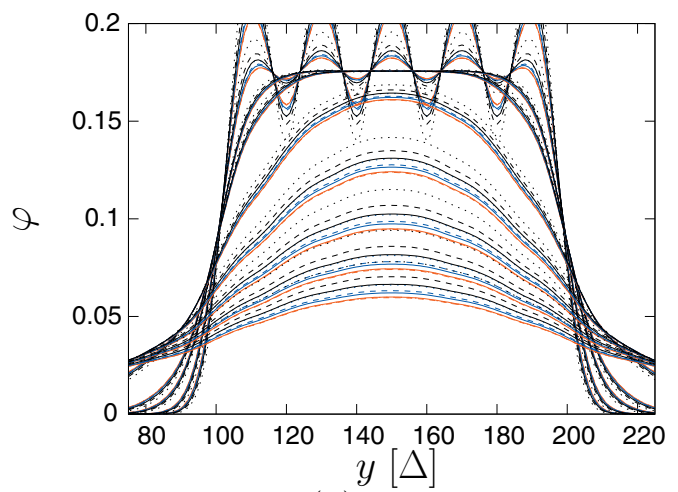

(b)

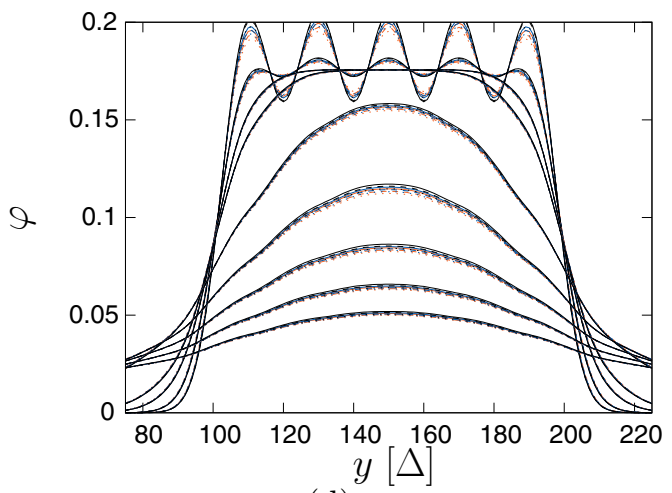

(d)

FIG. 5. Concentration distributions along the central pore channel of cubic matrix [the line $(150 \Delta, y)$ ] at various values of $\tau$. (a) linear and (c) nonlinear at $\tau=1,5,10,25,50,75,100,150,300$, (b) linear and (d) nonlinear at $\tau=150,300,500,2000,4000,6000,8000,10000$ using diffusion coefficients given in Table I. The legend is the same as in Fig. 1.

$$
\varphi_{\mathrm{ex}}(\tau)=\int_{0}^{\tau} \frac{\partial \varphi_{\mathrm{ex}}}{\partial t}\left(t^{\prime}\right) d t^{\prime}=\int_{0}^{\tau} d t^{\prime} \int_{S} \vec{j}\left(\vec{r}, t^{\prime}\right) \cdot d \vec{S}
$$

First we consider the case where the matrix particle size, pore volume, and total effective pore-opening area are kept fixed while varying the pore size and focus on the cubic pore symmetry and using the parameter set corresponding to $k$ $=2$. Three different pore sizes were, namely, $d, d / 3$, and $d / 7$, where $d$ was chosen to be $21 \Delta$. The resulting pore wall thickness (in order to assure equal matrix particle size, pore volume, and total effective pore-opening area) was $40 \Delta, 20 \Delta$, and $10 \Delta$, respectively. The thickness of the empty region surrounding the porous matrix particle was $50 \Delta$ in all three cases. Initially the concentration was set to be uniform throughout the matrix $(\varphi=1$ everywhere inside the porous matrix and 0 outside the matrix) and the total amount of substance was set to be equal in all cases. As seen from Fig. 6(b) (blue lines) the exit rate is fastest in the case of smaller pores for small $\tau$ values (from top to bottom the positions of curves correspond to $d / 7, d / 3$, and $d$ ) whereas, eventually, the situation reverses at some intermediate values of $\tau$ [inset of Fig. 6(b)]. The integral with respect to $\tau$ represents the fraction of solute outside the matrix and is given in Fig. 6(a). The latter is the largest in case of the smallest pore size in the whole time interval, but the curves eventually converge, which is due to equal system volumes. Although this might seem surprising at first sight, it is actually straightforward. Namely, the available volume outside of the matrix can be filled more efficiently if there are more sources present. The volume per unit pore-opening area into which the molecules can flow by means of lateral diffusion currents is larger in case of smaller pore size (which can be observed from the width/area of the concentration isosurfaces in Fig. 7, which are wider in case of smaller pores). The same holds for hexagonal symmetry. Recently, Van Speybroeck et al. ${ }^{2}$ observed that the transport rate of drug molecules from the SBA-15 matrix was in fact faster that in the case of pure drug particles which they attributed either to changes in the crystal structure and size of the confined drug particles. In their experiment they measured the concentration of dissolved drug molecules in the bulk aqueous suspensions (in some cases surfactant molecules were added to simulate physiological conditions) of various drug and drug loaded SBA particles, respectively, by means of UV spectrometry at different time intervals. Prior to measurements the withdrawn samples were filtered to remove solid particles. The model drugs were only poorly water soluble. Since the release is supposed to be controlled principally by means of diffusion, we wanted to explore the possibility that, besides the difference in the size of drug particles, the reason for increased release rate was purely geometrical. Therefore, we solved the diffusion Eq. (5) for exactly the same initial conditions using the same parameter set $k=2$ as above but in absence of the matrix walls. In experiment one measures the concentration in the bulk solution phase containing the matrix or drug particles. Therefore one measures the rate at which molecules diffuse across the diffusion-controlled liquid layer. The lateral diffusion currents within the diffusion layer in fact slow 


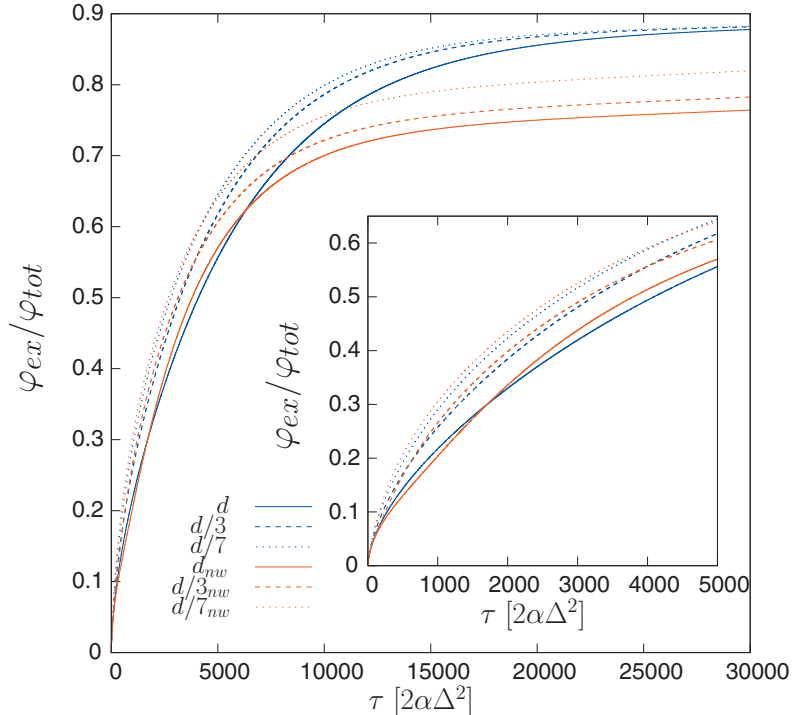

(a)

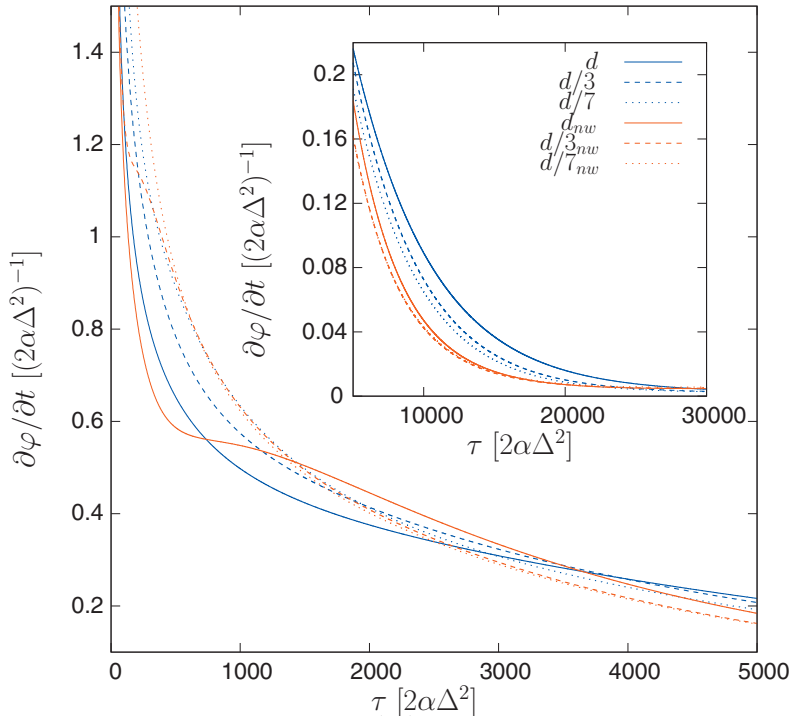

(b)

FIG. 6. (a) Total substance outside the matrix and (b) transfer rate across the outer matrix surface. Blue lines correspond to matrix systems and red lines to free diffusion (that is, the evolution of the system under the same initial conditions using the same parameter set $k$, but in the absence of pore walls). The surface, across which the rate is calculated, is equal in all cases.

down the overall diffusion across this layer. The thickness of this diffusion layer depends on the particle size, fluid properties, and mixing. Furthermore since polycrystalline drug particles are used with sizes of several tens of microns, whereas the size of SBA-type matrix particles is up to a few microns (i.e., drug particles and matrix particles have different sizes and thus different diffusion layer thickness), it is quite difficult to compare the results of experiments directly from the present theoretical point of view. A rather unambiguous manner to do so is to consider the transfer rate across the same surface as in the presence of matrix walls (that is, the initial solute concentration distribution is the same as in the presence of the porous matrix, but the system evolves freely in the absence of pore walls and the surface, across which the transfer rate is measured, remains the same as in the presence of the matrix) using the same parameter set $k=2$. The results are shown in Figs. 6(a) and 6(b) (red lines). The evolution of outgoing rates has two regimes. The one at small $\tau$ values is due to the transport at positions where the pore channels (however without the pore walls) are connected to the outer surface, whereas the second burst in transfer rate arises when the diffusion front of the inner molecules arrives at the surface. The time when the crossover between regimes occurs is longer in case of larger pore size which is readily observed in Fig. 6(b). The rate after the crossover is fastest in the case of largest pore size. As compared to matrix systems with corresponding equal pore sizes, the rates are initially slower for very low $\tau$ values, afterward they become faster and eventually again fall off bellow the values of corresponding matrix systems at longer $\tau$ values. The temporal distance between both intersecting points increases with increasing pore size. Initially the rate in the case of matrix systems is faster because there is no lateral diffusion and thus no initial dilution before exiting the outer surface. The first intersection arises when the molecules located further to the center reach the surface, while in the case of matrix systems the onset diffusion front still has not reached those molecules. However, due to the larger volume that is accessible to the diffusing molecules in the absence of pore walls, the system eventually becomes diluted internally and

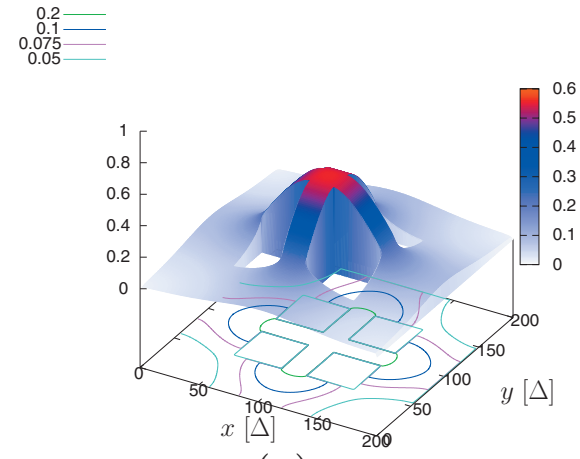

(a)

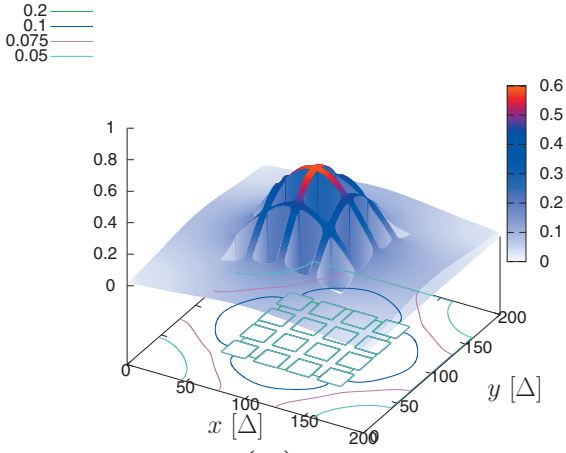

(b)

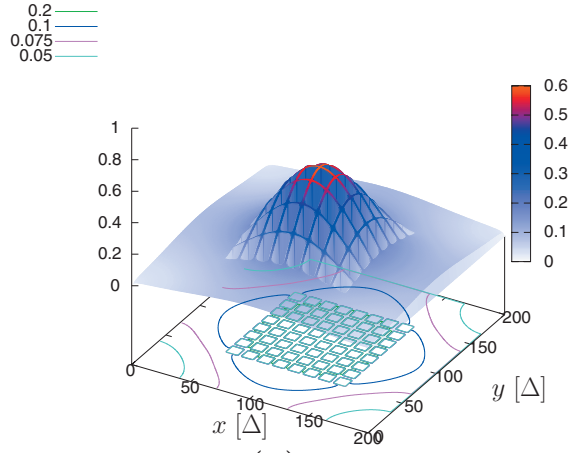

(c)

FIG. 7. Concentration fields in cubic porous systems at $\tau=1500$. The area of all concentration isosurfaces is larger in the case of smaller pore size. The increase is due to more effective diffusion (drainage) in the direction perpendicular to the surface normal. 


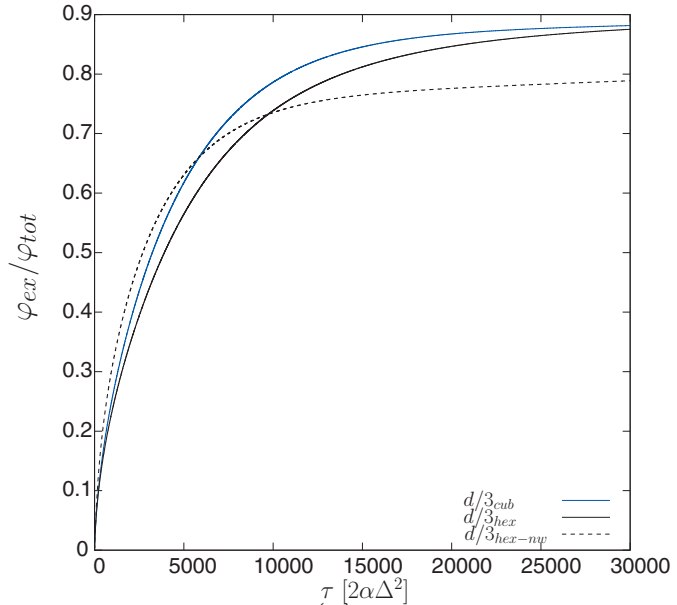

(a)

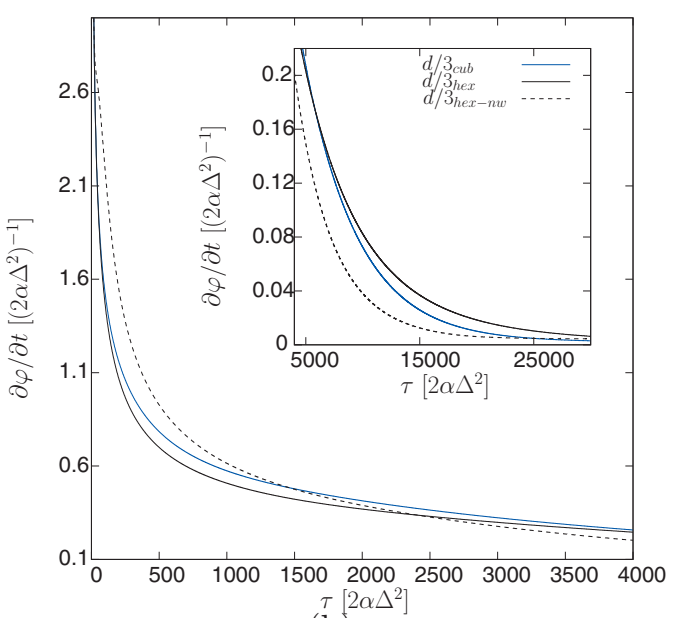

$(\mathrm{b})$

FIG. 8. (a) Total substance outside the matrix and (b) transfer rate across the outer matrix surface. Black lines correspond to hexagonal system and blue to corresponding cubic system with equal pore size. The broken line denotes the absence of matrix walls (free diffusion), i.e., the evolution of the system under the same initial conditions using the same parameter set $k$, but in the absence of pore walls. The surface, across which the rate is calculated, corresponds to the outer surface of the porous matrix particle.

the concentration gradient across the outer surface decreases. Although the effective surface through which the molecules can leave the matrix is smaller, the overall transfer rate eventually becomes faster due to higher gradients across the outer surface due to less internal dilution. The corresponding evolution of total amount of substance released through the outer surface also exhibits two intersections with corresponding curves of matrix systems and thus the total amount is a bit larger for a short intermediate time interval, after which it falls bellow again and stays at the same level. The lower final value is due to larger total volume of the system (recall that there are no walls present). Qualitatively the same conclusions hold for a matrix with hexagonal symmetry. Exact comparison of hexagonal and cubic symmetry is not possible because one cannot assure equal pore volume, pore size, and effective pore exit area at the same time. Therefore we choose to set the pore size equal to $d / 3$ and create a system with almost equal pore volume (the latter was $0.5 \%$ larger in case of hexagonal symmetry) by taking seven pore channels of equal width and taking one side of the square representing the outer matrix surface $6 \%$ shorter and the other side $1.3 \%$ shorter. The specific dimensions of the matrix particle with hexagonal pore structure were the following. The length and width of pore walls were $90 \Delta$ and $8 \Delta$ and the pore diameter was $7 \Delta$. The thickness of the empty region surrounding the porous matrix particle was again $50 \Delta$. As in the case of cubic symmetry, the evolution of the system under exactly the same initial conditions but in the absence of pore walls is denoted with the index $n w$. Such a system has $0.5 \%$ larger pore volume. The results together with the corresponding results of the cubic system are plotted in Fig. 8. The point at which the total release from the matrix becomes faster then free diffusion is shifted toward longer $\tau$ compared to cubic systems. Furthermore it is observed that the release from the cubic system with equal pore size and approximately equal pore volume is faster than from the hexagonal one. This indicates that besides pore volume, pore size, and matrixparticle size the transport also depends on the geometry of the porous network. As regards experimental findings, unfortunately, Van Speybroeck et al. did not sample short time intervals so that only long-time tails can be compared. Still, the relative profile shapes and differences between pure drug particles and matrix systems are reproduced quite nicely by our model, although we considered equal sizes of matrixloaded and free particles. Our analysis shows that the enhancement of drug release by incorporating drugs into porous matrices is mainly due to geometrical reasons. Note that among all substances studied by Van Speybroeck et al. they found exceptions where the release slowed down after the incorporation into the SBA-15 matrix, which they attributed to specific interactions between the drug molecules and the matrix walls. Naturally, such cases are not supported by the present model as it is, but can be incorporated by means of an external potential. In such a case the evolution of the system would be governed by free-energy gradients.

\section{CONCLUSION}

A mathematical model was developed to describe nonlinear diffusion in 2D ordered porous media on a continuum level. Basic molecular properties, such as molecular volume and generic vdW parameters, were introduced to the macroscopic diffusion equation through a modified free volume theory of binary diffusion coefficients. The resulting governing equation was solved numerically with a fully implicit ADI method. Our analysis shows that the nonlinear diffusion controlled release from an ordered porous matrix particle does not depend strongly on the molecular properties of the solute. In contrast it was also shown that simple linear diffusion predicts a significant influence of molecular parameters on diffusion inside and from the porous matrix particle. Somewhat surprisingly, the release is enhanced with respect to the release from free particles in absence of pore walls. This finding coincides well with the recent experimental results from drug release studies and indicates that the enhanced release could be mainly due to geometrical reasons. 
Further, the present results predict that the release depends on pore size, volume, and pore symmetry. For example, it is faster in the case of smaller pore sizes assuming equal pore volume and, also, it is faster in the case of cubic symmetry, if assuming equal pore size and volume. The latter reveals the actual underlying complexity of the relationship between the diffusion kinetics and the matrix geometry and its parameters. In this respect, the developed model along with further detailed experimental studies could be useful for optimization of matrix parameters in order to obtain desired release profiles.

\section{ACKNOWLEDGMENTS}

This work was supported by the Ministry of Higher Education, Science and Technology of the Republic of Slovenia under Grant Nos. P1-0002 and J1-9804.

\section{APPENDIX A: GENERIC VDW EQUATION OF STATE}

The generic (or virial) vdW equation of state for a mixture of $c$ constituents with number densities $\rho_{n}, n=1, \cdots c$ and pair interaction potentials $u_{i j}(r)$ at temperature $T$ and total number density $\rho$ is written as

$$
\begin{aligned}
\frac{\beta p}{\rho}= & 1-\frac{2 \pi}{3 \rho} \beta \sum_{i=1}^{c} \sum_{j=1}^{c} \rho_{i} \rho_{j} \int_{0}^{\infty} d r r^{3} \frac{d u_{i j}}{d r} \\
& \times \exp \left[-\beta u_{i j}\right] y_{i j}\left(r ; \rho_{i}, T\right),
\end{aligned}
$$

where $\rho_{n}$ denotes the set of constituent number densities $\rho_{n}, n=1, \cdots c, \beta=1 / k T$, and $y_{i j}\left(r ; \rho_{i}, T\right)$ is the cavity function, given by the usual relation

$$
y_{i j}\left(r ; \rho_{i}, T\right)=\exp \left[\beta u_{i j}\right] g_{i j}\left(r ; \rho_{i}, T\right),
$$

where $g_{i j}\left(r ; \rho_{i}, T\right)$ stands for the equilibrium pair correlation function. Realistic pair potentials consist of an attractive and a repulsive part and the interparticle distance at zero of pair potential energy can be denoted as $\sigma_{i j}\left(u_{i j}\left(\sigma_{i j}\right)=0\right)$. The integral in Eq. (A1) can be split in two parts (a repulsive part $0<r<\sigma_{i j}$ and an attractive part $r>\sigma_{i j}$ ) and the resulting equation rearranged into the form of the classical vdW equation of state, with generic parameters $\mathrm{A}$ and $\mathrm{B}$ given by

$$
\begin{aligned}
& A=\sum_{i=1}^{c} \sum_{j=1}^{c} A_{i j} X_{i} X_{j}, \\
& B=\frac{\sum_{i=1}^{c} \sum_{j=1}^{c} B_{i j} X_{i} X_{j}}{1+\rho \sum_{i=1}^{c} \sum_{j=1}^{c} B_{i j} X_{i} X_{j}},
\end{aligned}
$$

where

$$
\begin{aligned}
& A_{i j}=\frac{2 \pi}{3} \int_{\sigma_{i j}}^{\infty} d r r^{3} \frac{d u_{i j}}{d r} g_{i j}(r), \\
& B_{i j}=\frac{2 \pi}{3} \beta \int_{0}^{\sigma_{i j}} d r r^{3} \frac{d u_{i j}}{d r} g_{i j}(r) .
\end{aligned}
$$

$\sigma_{i j}$ thus stands for a characteristic size parameter, and is influenced by both repulsive and attractive interactions. For a two component fluid the generic parameter $B$ is given by

$$
B=\frac{1}{C}\left(B_{11} X_{1}^{2}+B_{22} X_{2}^{2}+X_{1} X_{2}\left[B_{12}+B_{21}\right]\right),
$$

where the denominator of Eq. (A4) (essentially a constant for a given system at given temperature and density) has been absorbed in the constant $c$. The only difference between parameters $B_{12}$ and $B_{21}$ is in the pair correlation function and they become equal in the limit of vanishing density. The sum $B_{12}+B_{21}$ can be thought of as a common cross coefficient $2 B_{12}^{c}$ used in Eq. (2). Furthermore if the constituents have similar properties $\left(\sigma_{i i} \approx \sigma_{j j} \approx \sigma_{i j}\right.$, and similarly for the depths of potential minima) we can use combining rules for the cross coefficient $B_{12}+B_{21}=2 \sqrt{B_{11} B_{22}}$. This approximation has to be made in order to arrive at an expression for the diffusion coefficient which can be used directly in the diffusion equation. We now turn to the validity of these approximations as a function of the mole fractions of constituents. Ben-Naim has shown ${ }^{26}$ that the heights and positions of the first peak (positioned roughly at $\sigma_{i j}$ ) of pair correlation functions of binary LJ mixtures almost do not change when one varies the mole fraction of one component from 0 to 1 . The position and height of the second peaks, however, depend on the composition of the mixture. Since the integral in Eq. (A6) goes from 0 to $\sigma_{i j}$ the approximations should therefore hold for all compositions.

\section{APPENDIX B: BOUNDARY CONDITIONS USED IN THE CALCULATION}

Neumann boundary conditions are used (zero gradient) and the resulting formulae are presented according to the classification of points on the grid:

(1) Points neighboring the left outer boundary and points directly at right pore walls: $\varphi_{i-1, j}=\varphi_{i, j}$ and $\psi_{i-1, j}=\psi_{i, j}$,

$$
\begin{aligned}
{[1+} & \left.\alpha D\left(\varphi_{i, j}^{n}\right)\right] \varphi_{i, j}^{n+1 / 2}-\alpha D\left(\varphi_{i+1, j}^{n}\right) \varphi_{i+1, j}^{n+1 / 2} \\
= & \alpha\left(\psi_{i, j-1}^{n}-3 \psi_{i, j}^{n}+\psi_{i+1, j}^{n}+\psi_{i, j+1}^{n}\right. \\
& \left.+\left[1 / \alpha+D\left(\varphi_{i, j}^{n}\right)\right] \varphi_{i, j}^{n}-D\left(\varphi_{i+1, j}^{n}\right) \varphi_{i+1, j}^{n}\right), \\
-\alpha D\left(\varphi_{i, j-1}^{n+1 / 2}\right) \varphi_{i, j-1}^{n+1}+\left[1+2 \alpha D\left(\varphi_{i, j}^{n+1 / 2}\right)\right] \varphi_{i, j}^{n+1} & -\alpha D\left(\varphi_{i, j+1}^{n+1 / 2}\right) \varphi_{i, j+1}^{n+1} \\
& -\alpha\left(\psi_{i, j-1}^{n+1 / 2}-3 \psi_{i, j}^{n+1 / 2}+\psi_{i+1, j}^{n+1 / 2}+\psi_{i, j+1}^{n+1 / 2}\right. \\
& -D\left(\varphi_{i, j-1}^{n+1 / 2}\right) \varphi_{i, j-1}^{n+1 / 2}+\left[1 / \alpha+2 D\left(\varphi_{i, j}^{n+1 / 2}\right)\right] \varphi_{i, j}^{n+1 / 2} \\
& \left.-D\left(\varphi_{i, j+1}^{n+1 / 2}\right) \varphi_{i, j+1}^{n+1 / 2}\right) .
\end{aligned}
$$

(2) Points neighboring the right outer boundary and points directly at left pore walls: $\varphi_{i+1, j}=\varphi_{i, j}$ and $\psi_{i+1, j}=\psi_{i, j}$,

$$
\begin{aligned}
-\alpha & D\left(\varphi_{i-1, j}^{n}\right) \varphi_{i-1, j}^{n+1 / 2}+\left[1+\alpha D\left(\varphi_{i, j}^{n}\right)\right] \varphi_{i, j}^{n+1 / 2} \\
= & \alpha\left(\psi_{i-1, j}^{n}+\psi_{i, j-1}^{n}-3 \psi_{i, j}^{n}+\psi_{i, j+1}^{n}-D\left(\varphi_{i-1, j}^{n}\right) \varphi_{i-1, j}^{n}\right. \\
& \left.+\left[1 / \alpha+D\left(\varphi_{i, j}^{n}\right)\right] \varphi_{i, j}^{n}\right)
\end{aligned}
$$




$$
\begin{aligned}
-\alpha D & \left(\varphi_{i, j-1}^{n+1 / 2}\right) \varphi_{i, j-1}^{n+1}+\left[1+2 \alpha D\left(\varphi_{i, j}^{n+1 / 2}\right)\right] \varphi_{i, j}^{n+1} \\
& -\alpha D\left(\varphi_{i, j+1}^{n+1 / 2}\right) \varphi_{i, j+1}^{n+1} \\
= & \alpha\left(\psi_{i-1, j}^{n+1 / 2}+\psi_{i, j-1}^{n+1 / 2}-4 \psi_{i, j}^{n+1 / 2}+\psi_{i+1, j}^{n+1 / 2}+\psi_{i, j+1}^{n+1 / 2}\right. \\
& -D\left(\varphi_{i, j-1}^{n+1 / 2}\right) \varphi_{i, j-1}^{n+1 / 2}+\left[1 / \alpha+2 D\left(\varphi_{i, j}^{n+1 / 2}\right)\right] \varphi_{i, j}^{n+1 / 2} \\
& \left.-D\left(\varphi_{i, j+1}^{n+1 / 2}\right) \varphi_{i, j+1}^{n+1 / 2}\right) .
\end{aligned}
$$

(3) Points neighboring the bottom outer boundary and points directly at top pore walls: $\varphi_{i, j-1}=\varphi_{i, j}$ and $\psi_{i, j-1}=\psi_{i, j}$

$$
\begin{aligned}
-\alpha D & \left(\varphi_{i-1, j}^{n}\right) \varphi_{i-1, j}^{n+1 / 2}+\left[1+2 \alpha D\left(\varphi_{i, j}^{n}\right)\right] \varphi_{i, j}^{n+1 / 2} \\
& -\alpha D\left(\varphi_{i+1, j}^{n}\right) \varphi_{i+1, j}^{n+1 / 2} \\
= & \alpha\left(\psi_{i-1, j}^{n}-3 \psi_{i, j}^{n}+\psi_{i+1, j}^{n}+\psi_{i, j+1}^{n}-D\left(\varphi_{i-1, j}^{n}\right) \varphi_{i-1, j}^{n}\right. \\
& \left.+\left[1 / \alpha+2 D\left(\varphi_{i, j}^{n}\right)\right] \varphi_{i, j}^{n}-D\left(\varphi_{i+1, j}^{n}\right) \varphi_{i+1, j}^{n}\right),
\end{aligned}
$$

$$
\begin{aligned}
{[1+} & \left.\alpha D\left(\varphi_{i, j}^{n+1 / 2}\right)\right] \varphi_{i, j}^{n+1}-\alpha D\left(\varphi_{i, j+1}^{n+1 / 2}\right) \varphi_{i, j+1}^{n+1} \\
= & \alpha\left(\psi_{i-1, j}^{n+1 / 2}-3 \psi_{i, j}^{n+1 / 2}+\psi_{i+1, j}^{n+1 / 2}+\psi_{i, j+1}^{n+1 / 2}\right. \\
& \left.+\left[1 / \alpha+D\left(\varphi_{i, j}^{n+1 / 2}\right)\right] \varphi_{i, j}^{n+1 / 2}-D\left(\varphi_{i, j+1}^{n+1 / 2}\right) \varphi_{i, j+1}^{n+1 / 2}\right) .
\end{aligned}
$$

(4) Points neighboring the top outer boundary and points directly at bottom pore walls: $\varphi_{i, j+1}=\varphi_{i, j}$ and $\psi_{i, j+1}=\psi_{i, j}$,

$$
\begin{aligned}
-\alpha & \left(\varphi_{i-1, j}^{n}\right) \varphi_{i-1, j}^{n+1 / 2}+\left[1+2 \alpha D\left(\varphi_{i, j}^{n}\right)\right] \varphi_{i, j}^{n+1 / 2} \\
& -\alpha D\left(\varphi_{i+1, j}^{n}\right) \varphi_{i+1, j}^{n+1 / 2} \\
= & \alpha\left(\psi_{i-1, j}^{n}+\psi_{i, j-1}^{n}-3 \psi_{i, j}^{n}+\psi_{i+1, j}^{n}-D\left(\varphi_{i-1, j}^{n}\right) \varphi_{i-1, j}^{n}\right. \\
& \left.+\left[1 / \alpha+D\left(\varphi_{i, j}^{n}\right)\right] \varphi_{i, j}^{n}-D\left(\varphi_{i+1, j}^{n}\right) \varphi_{i+1, j}^{n}\right),
\end{aligned}
$$

$$
\begin{aligned}
-\alpha & \left(\varphi_{i, j-1}^{n+1 / 2}\right) \varphi_{i, j-1}^{n+1}+\left[1+\alpha D\left(\varphi_{i, j}^{n+1 / 2}\right)\right] \varphi_{i, j}^{n+1} \\
= & \alpha\left(\psi_{i-1, j}^{n+1 / 2}+\psi_{i, j-1}^{n+1 / 2}-3 \psi_{i, j}^{n+1 / 2}+\psi_{i+1, j}^{n+1 / 2}\right. \\
& \left.-D\left(\varphi_{i, j-1}^{n+1 / 2}\right) \varphi_{i, j-1}^{n+1 / 2}+\left[1 / \alpha+D\left(\varphi_{i, j}^{n+1 / 2}\right)\right] \varphi_{i, j}^{n+1 / 2}\right) .
\end{aligned}
$$

(5) Top left corner: $\varphi_{i-1, j}=\varphi_{i, j}$ and $\psi_{i, j+1}=\psi_{i, j}$,

$$
\begin{aligned}
{[1+} & \left.\alpha D\left(\varphi_{i, j}^{n}\right)\right] \varphi_{i, j}^{n+1 / 2}-\alpha D\left(\varphi_{i+1, j}^{n}\right) \varphi_{i+1, j}^{n+1 / 2} \\
= & \alpha\left(\psi_{i, j-1}^{n}-2 \psi_{i, j}^{n}+\psi_{i+1, j}^{n}+\left[1 / \alpha+D\left(\varphi_{i, j}^{n}\right)\right] \varphi_{i, j}^{n}\right. \\
& \left.-D\left(\varphi_{i+1, j}^{n}\right) \varphi_{i+1, j}^{n}\right), \\
-\alpha & D\left(\varphi_{i, j-1}^{n+1 / 2}\right) \varphi_{i, j-1}^{n+1}+\left[1+\alpha D\left(\varphi_{i, j}^{n+1 / 2}\right)\right] \varphi_{i, j}^{n+1} \\
= & \alpha\left(\psi_{i, j-1}^{n+1 / 2}-2 \psi_{i, j}^{n+1 / 2}+\psi_{i+1, j}^{n+1 / 2}-D\left(\varphi_{i, j-1}^{n+1 / 2}\right) \varphi_{i, j-1}^{n+1 / 2}\right. \\
& \left.+\left[1 / \alpha+D\left(\varphi_{i, j}^{n+1 / 2}\right)\right] \varphi_{i, j}^{n+1 / 2}\right) .
\end{aligned}
$$

(6) Bottom left corner: $\varphi_{i-1, j}=\varphi_{i, j}$ and $\psi_{i, j-1}=\psi_{i, j}$,

$$
\begin{aligned}
{[1+} & \left.\alpha D\left(\varphi_{i, j}^{n}\right)\right] \varphi_{i, j}^{n+1 / 2}-\alpha D\left(\varphi_{i+1, j}^{n}\right) \varphi_{i+1, j}^{n+1 / 2} \\
= & \alpha\left(\psi_{i, j+1}^{n}-2 \psi_{i, j}^{n}+\psi_{i+1, j}^{n}+\left[1 / \alpha+D\left(\varphi_{i, j}^{n}\right)\right] \varphi_{i, j}^{n}\right. \\
& \left.-D\left(\varphi_{i+1, j}^{n}\right) \varphi_{i+1, j}^{n}\right),
\end{aligned}
$$

$$
\begin{aligned}
-\alpha & D\left(\varphi_{i, j+1}^{n+1 / 2}\right) \varphi_{i, j+1}^{n+1}+\left[1+\alpha D\left(\varphi_{i, j}^{n+1 / 2}\right)\right] \varphi_{i, j}^{n+1} \\
= & \alpha\left(\psi_{i, j-1}^{n+1 / 2}-2 \psi_{i, j}^{n+1 / 2}+\psi_{i+1, j}^{n+1 / 2}-D\left(\varphi_{i, j+1}^{n+1 / 2}\right) \varphi_{i, j+1}^{n+1 / 2}\right. \\
& \left.+\left[1 / \alpha+D\left(\varphi_{i, j}^{n+1 / 2}\right)\right] \varphi_{i, j}^{n+1 / 2}\right) .
\end{aligned}
$$

(7) Bottom right corner: $\varphi_{i+1, j}=\varphi_{i, j}$ and $\psi_{i, j-1}=\psi_{i, j}$,

$$
\begin{aligned}
-\alpha & D\left(\varphi_{i-1, j}^{n}\right) \varphi_{i-1, j}^{n+1 / 2}+\left[1+\alpha D\left(\varphi_{i, j}^{n}\right)\right] \varphi_{i, j}^{n+1 / 2} \\
= & \alpha\left(\psi_{i-1, j}^{n}-2 \psi_{i, j}^{n}+\psi_{i, j+1}^{n}-D\left(\varphi_{i-1, j}^{n}\right) \varphi_{i-1, j}^{n}\right. \\
& \left.+\left[1 / \alpha+D\left(\varphi_{i, j}^{n}\right)\right] \varphi_{i, j}^{n}\right), \\
& {\left[1+\alpha D\left(\varphi_{i, j}^{n+1 / 2}\right)\right] \varphi_{i, j}^{n+1}-\alpha D\left(\varphi_{i, j+1}^{n+1 / 2}\right) \varphi_{i, j+1}^{n+1} } \\
= & \alpha\left(\psi_{i-1, j}^{n+1 / 2}-2 \psi_{i, j}^{n+1 / 2}+\psi_{i, j+1}^{n+1 / 2}\right. \\
& \left.+\left[1 / \alpha+D\left(\varphi_{i, j}^{n+1 / 2}\right)\right] \varphi_{i, j}^{n+1 / 2}-D\left(\varphi_{i, j+1}^{n+1 / 2}\right) \varphi_{i, j+1}^{n+1 / 2}\right) .
\end{aligned}
$$

(8) Top right corner: $\varphi_{i+1, j}=\varphi_{i, j}$ and $\psi_{i, j+1}=\psi_{i, j}$,

$$
\begin{aligned}
-\alpha & D\left(\varphi_{i-1, j}^{n}\right) \varphi_{i-1, j}^{n+1 / 2}+\left[1+\alpha D\left(\varphi_{i, j}^{n}\right)\right] \varphi_{i, j}^{n+1 / 2} \\
= & \alpha\left(\psi_{i-1, j}^{n}-2 \psi_{i, j}^{n}+\psi_{i, j-1}^{n}-D\left(\varphi_{i-1, j}^{n}\right) \varphi_{i-1, j}^{n}\right. \\
& \left.+\left[1 / \alpha+D\left(\varphi_{i, j}^{n}\right)\right] \varphi_{i, j}^{n}\right), \\
{[1+} & \left.\alpha D\left(\varphi_{i, j}^{n+1 / 2}\right)\right] \varphi_{i, j}^{n+1}-\alpha D\left(\varphi_{i, j-1}^{n+1 / 2}\right) \varphi_{i, j-1}^{n+1} \\
= & \alpha\left(\psi_{i-1, j}^{n+1 / 2}-2 \psi_{i, j}^{n+1 / 2}+\psi_{i, j-1}^{n+1 / 2}\right. \\
& \left.+\left[1 / \alpha+D\left(\varphi_{i, j}^{n+1 / 2}\right)\right] \varphi_{i, j}^{n+1 / 2}-D\left(\varphi_{i, j-1}^{n+1 / 2}\right) \varphi_{i, j-1}^{n+1 / 2}\right) .
\end{aligned}
$$

The program identifies the point type (ordinary point or a specific type of boundary point given above) and, according to the direction currently being updated, constructs the tridiagonal matrix $\mathbf{T}$ and vector $\vec{r}$ (see main text) and the resulting linear equations are solved with $L U$ decomposition, forward and backward substitution.

${ }^{1}$ F. Hoffmann, M. Cornelius, J. Morell, and M. Fröba, Angew. Chem., Int. Ed. 45, 3216 (2006).

${ }^{2}$ M. Vallet-Regí, F. Balas, and D. Arcos, Angew. Chem., Int. Ed. 46, 7548 (2007); M. Vallet-Regí, L. Ruiz-González, I. Izquierdo-Barba, and J. M. González-Calbet, J. Mater. Chem. 16, 26 (2006); M. Van Speybroeck, V. Barillaro, T. D. Thi, R. Mellaerts, J. Martens, J. Van Humbeeck, J. Vermant, P. Annaert, G. Van der Mooter, and P. Augustijns, J. Pharm. Sci. 98, 2648 (2009).

${ }^{3}$ D. Stauffer, Phys. Rep. 54, 1 (1979)

${ }^{4}$ S. Havlin and D. Ben-Avraham, Adv. Phys. 51, 187 (2002).

${ }^{5}$ T. Nakayama, K. Yakubo, and R. L. Orbach, Rev. Mod. Phys. 66, 381 (1994).

${ }^{6}$ B. J. Sung and A. Yethiray, J. Chem. Phys. 128, 054702 (2008).

${ }^{7}$ A. K. Chakraborty, D. Bratko, and D. Chandler, J. Chem. Phys. 100, 1528 (1994)

${ }^{8}$ H. Dominguez and M. Rivera, J. Chem. Phys. 123, 066101 (2005).

${ }^{9}$ F. Höfling, T. Franosch, and E. Frey, Phys. Rev. Lett. 96, 165901 (2006).

${ }^{10}$ R. Villalobos, S. Cordero, A. M. Vidales, and A. Dominguez, Physica A 367, 305 (2006).

${ }^{11}$ K. Kosmidis and P. Argyrakis, J. Chem. Phys. 119, 6373 (2003).

${ }^{12}$ B. I. Halperin, S. Feng, and P. N. Sen, Phys. Rev. Lett. 54, 2391 (1985).

${ }^{13}$ J. L. Vásquez, "Perspectives in nonlinear diffusion: between analysis, physics and geometry," Proceedings of the International Congress of Mathematicians, Eur. Math. Soc., Madrid, Spain, 2006 (unpublished).

${ }^{14}$ G. M. Laudone, G. P. Matthews, and P. A. C. Gane, Chem. Eng. Sci. 63, 1987 (2008) 
${ }^{15}$ V. Lemaire, J. Bélair, and P. Hildgen, Int. J. Pharm. 258, 95 (2003).

${ }^{16}$ K. Rah and B. C. Eu, J. Chem. Phys. 116, 7967 (2002).

${ }^{17}$ J. Crank, The Mathematics of Diffusion (Clarendon, Oxford, 1975).

${ }^{18}$ P. S. Burada, P. Hanggi, H. C. Mult, F. Marchesoni, G. Schmid, and P. Talkner, ChemPhysChem 10, 45 (2009).

${ }^{19}$ Y. Sakamoto, M. Kaneda, O. Terasaki, D. Y. Zhao, J. M. Kim, G. Stucky, H. J. Shin, and R. Ryoo, Nature (London) 408, 449 (2000).

${ }^{20}$ M. H. Cohen and D. Turnbull, J. Chem. Phys. 31, 1164 (1959).

${ }^{21}$ K. Rah and B. C. Eu, J. Chem. Phys. 115, 2634 (2001).

${ }^{22}$ S. Chapman and T. G. Cowling, The Mathematical Theory of Nonuniform
Gases (Cambridge University Press, London, 1970).

${ }^{23}$ J. Vidal, Thermodynamique Pétrolière (Technip, Paris, 1997).

${ }^{24}$ W. H. Press, S. A. Teukolsky, W. T. Vetterling, and B. P. Flannery, Numerical Recipes: The Art of Scientific Computing, 3rd ed. (Cambridge University Press, New York, 2007).

${ }^{25}$ G. A. Mansoori, N. F. Carnahan, and K. E. Starling, J. Chem. Phys. 54, 1523 (1971).

${ }^{26}$ A. Ben-Naim, Water and Aqueous Solutions (Plenum, New York, 1979); A. Ben-Naim, Molecular Theory of Solutions (Oxford University Press, New York, 2006). 